\title{
Diversity soliton solutions for the (2+1)-Dimensional Boiti-Leon-Manna-Pempinelli Equation
}

\author{
Xichao Deng ${ }^{1}$, Hanlin Chen $^{2} \&$ Zhenhui $\mathrm{Xu}^{3}$ \\ ${ }^{1}$ Applied Technology College, Southwest University of Science and Technology, Mianyang 621010, PR China \\ ${ }^{2}$ School of Science, Southwest University of Science and Technology, Mianyang 621010, PR China. \\ ${ }^{3}$ Applied Technology College, Southwest University of Science and Technology, Mianyang 621010, PR China \\ Correspondence: Zhenhui Xu, Applied Technology College, Southwest University of Science and Technology, \\ Mianyang 621010, PR China. Tel: 86-13458302193 E-mail: xuzhenhui19@163.com
}

Received: September 15, 2014 Accepted: October 8, 2014 Online Published: October 28, 2014

doi:10.5539/jmr.v6n4p85 URL: http://dx.doi.org/10.5539/jmr.v6n4p85

This work was supported by Chinese Natural Science Foundation Grant No.11061028, Sichuan Educational science Foundation Grant No.09zc008.

\begin{abstract}
Diversity soliton solutions, including breather-type kink two wave solutions, cross-kink two solitary solutions, breather-type kink three wave solutions, kink three soliton solutions, are obtained for the $(2+1)$-Dimensional Boiti-Leon-Manna-Pempinelli Equation by using Hirota's bilinear form and extended homoclinic test approach, respectively. Moreover, the properties for some new solutions are shown with some figures.
\end{abstract}

Keywords: Boiti-Leon-Manna-Pempinelli Equation, extended homoclinic test approach, bilinear form, breathertype soliton, cross-kink two-soliton

\section{Introduction}

It is well known that exact solution of nonlinear evolution equations play an important role in nonlinear science fields, especially in nonlinear physical science since they can provide much physical information and more insight into the physical aspects of the problem and thus lead to further applications. Many effective and powerful methods to seek exact solutions were proposed, such as the inverse scattering method, the homogeneous balance method, Hirotas bilinear method, the Darboux transformation method, Wronskian technique and so on [1-6]. Very recently, Dai et al.[7-9] proposed a new technique called "the extended homoclinic test approach" to seek solitary wave solutions for integrable equations, and this method has been used to investigate several equations[10-11]. The extended homoclinic test technique is an extension of the homoclinic test method, the main difference between the two methods mentioned above is the test function of constructing exact solution.

In this paper, we consider (2+1)-Dimensional Boiti-Leon-Manna-Pempinelli Equation:

$$
\varphi_{y t}-3 \varphi_{x} \varphi_{x y}-3 \varphi_{y} \varphi_{x x}+\varphi_{x x x y}=0
$$

where $\varphi(x, y, t): R x \times R y \times R t \rightarrow R$ is a real function. It is called potential Boiti-Leon-Manna-Pempinelli (BLMP) equation. The Painlevé analysis, lax pairs and some exact solutions of Eq. (1) were given in Ref[12-13]. In this work, we mainly apply extended homoclinic test approach to determine diversity soliton solutions for Eq.(1). As a result, breather-type kink two wave solutions, cross-kink two solitary solutions and kink three soliton solutions are obtained.

By using Painlevé analysis, we assume

$$
\varphi=-2(\ln f)_{x},
$$

where $f=f(x, y, t)$ is unknown real function. Substituting Eq.(2) into Eq.(1) and using the bilinear form, we have

$$
\left(D_{y} D_{t}+D_{x}^{3} D_{y}\right) f \cdot f=0,
$$


where the bilinear operator $D$ is defined as

$$
D_{x}^{m} D_{y}^{n} D_{t}^{s} f(x, y, t) \cdot g(x, y, t)=\left.\left(\frac{\partial}{\partial x}-\frac{\partial}{\partial x^{\prime}}\right)^{m}\left(\frac{\partial}{\partial y}-\frac{\partial}{\partial y^{\prime}}\right)^{n}\left(\frac{\partial}{\partial t}-\frac{\partial}{\partial t^{\prime}}\right)^{s}\left[f(x, y, t) g\left(x^{\prime}, y^{\prime}, t^{\prime}\right)\right]\right|_{x^{\prime}=x, y^{\prime}=y, t^{\prime}=t} .
$$

\section{Breather-type Kink and Kink Two Wave Solutions}

Now we choose extended homoclinic test function

$$
f(x, y, t)=e^{-\theta}+\xi_{1} \cos (\tau)+\xi_{2} e^{\theta},
$$

where $\theta=\alpha_{1} x+\beta_{1} y+\gamma_{1} t, \tau=\alpha_{2} x+\beta_{2} y+\gamma_{1} t$ and $\alpha_{i}, \beta_{i}, \gamma_{i}, \xi_{i}(i=1,2)$ are some constants to be determined later. Substituting Eq.(4) into Eq.(3) and equating all the coefficients of different powers of $e^{\theta}, e^{-\theta}, \sin (\tau), \cos (\tau)$ and constant term to zero yields a set of algebraic equations:

$$
\left\{\begin{array}{l}
\xi_{1}\left(\beta_{1} \gamma_{1}-3 \alpha_{1}^{2} \alpha_{2} \beta_{2}-3 \alpha_{1} \alpha_{2}^{2} \beta_{1}-\beta_{2} \gamma_{2}+\alpha_{2}^{3} \beta_{2}+\alpha_{1}^{3} \beta_{1}\right)=0 \\
\xi_{1} \xi_{2}\left(\beta_{1} \gamma_{1}-3 \alpha_{1}^{2} \alpha_{2} \beta_{2}-3 \alpha_{1} \alpha_{2}^{2} \beta_{1}-\beta_{2} \gamma_{2}+\alpha_{2}^{3} \beta_{2}+\alpha_{1}^{3} \beta_{1}\right)=0 \\
\xi_{1} \xi_{2}\left(3 \alpha_{1}^{2} \alpha_{2} \beta_{1}-\alpha_{2}^{3} \beta_{1}+\alpha_{1}^{3} \beta_{2}-3 \alpha_{1} \alpha_{2}^{2} \beta_{2}+\beta_{2} \gamma_{1}+\beta_{1} \gamma_{2}\right)=0 \\
\xi_{1}\left(3 \alpha_{1}^{2} \alpha_{2} \beta_{1}-\alpha_{2}^{3} \beta_{1}+\alpha_{1}^{3} \beta_{2}-3 \alpha_{1} \alpha_{2}^{2} \beta_{2}+\beta_{2} \gamma_{1}+\beta_{1} \gamma_{2}\right)=0 \\
16 \alpha_{1}^{3} \beta_{1} \xi_{2}+4 \beta_{1} \gamma_{1} \xi_{2}-\beta_{2} \gamma_{2} \xi_{1}^{2}+4 \alpha_{2}^{3} \beta_{2} \xi_{1}^{2}=0 .
\end{array}\right.
$$

Solving the system of Eqs.(5), we obtain the following cases

Case(I):

$$
\alpha_{2}=-\frac{4 \xi_{2} \alpha_{1} \beta_{1}}{\xi_{1}^{2} \beta_{2}}, \gamma_{1}=\frac{\alpha_{1}^{3}\left(48 \xi_{2}^{2} \beta_{1}^{2}-\xi_{1}^{4} \beta_{2}^{2}\right)}{\xi_{1}^{4} \beta_{2}^{2}}, \gamma_{2}=\frac{4 \xi_{2} \alpha_{1}^{3} \beta_{1}\left(3 \xi_{1}^{4} \beta_{2}^{2}-16 \xi_{2}^{2} \beta_{1}^{2}\right)}{\xi_{1}^{6} \beta_{2}^{3}},
$$

where $\alpha_{1}, \beta_{1}, \beta_{2}, \xi_{1}, \xi_{2}$ are free real constants. Substituting Eq. (6) into Eq. (4) and taking $\xi_{2}>0, \xi_{1} \neq 0, \beta_{2} \neq 0$, we have

$$
f_{1}=2 \sqrt{\xi_{2}} \cosh \left(\alpha_{1} x+\beta_{1} y+E_{1} t+\frac{1}{2} \ln \left(\xi_{2}\right)\right)+\xi_{1} \cos \left(I_{1} x-\beta_{2} y+G_{1} t\right),
$$

where $E_{1}=\frac{\alpha_{1}^{3}\left(48 \xi_{2}^{2} \beta_{1}^{2}-\xi_{1}^{4} \beta_{2}^{2}\right)}{\xi_{1}^{4} \beta_{2}^{2}}, I_{1}=\frac{4 \xi_{2} \alpha_{1} \beta_{1}}{\xi_{1}^{2} \beta_{2}}, G_{1}=\frac{4 \xi_{2} \alpha_{1}^{3} \beta_{1}\left(3 \xi_{1}^{4} \beta_{2}^{2}-16 \xi_{2}^{2} \beta_{1}^{2}\right)}{\xi_{1}^{6} \beta_{2}^{3}}$. Substituting Eq. (7) into Eq. (2), we obtain the breather-type kink two-wave solutions for BLMP equation as follows(see Fig (a))

$$
\varphi_{1}=\frac{2 \xi_{2} \beta_{1} \sin (\tau)-4 \alpha_{1}\left(\xi_{1} \beta_{2} \sqrt{\xi_{2}} \sinh \left(\theta+\frac{1}{2} \ln \left(\xi_{2}\right)\right)\right)}{\xi_{1} \beta_{2}\left(2 \sqrt{\xi_{2}} \cosh \left(\theta+\frac{1}{2} \ln \left(\xi_{2}\right)\right)\right)+\xi_{1} \cos (\tau)},
$$

where $\theta=\alpha_{1} x+\beta_{1} y+E_{1} t, \tau=I_{1} x-\beta_{2} y+G_{1} t$. The solution represented by Eq.(8) is a breather-type kink two-wave which has breather effect when wave along with straight line $I_{1} x-\beta_{2} y+G_{1} t=d$ and it also is a kink solitary wave as it along with straight line $I_{1} x-\beta_{2} y+G_{1} t=d$, where $d$ are constants.

Case(II):

$$
\beta_{1}=\beta_{2} i, \gamma_{1}=-3 \alpha_{2}^{2} \alpha_{2} i+3 \alpha_{2}^{2} \alpha_{1}-\alpha_{1}^{3}+\alpha_{2}^{3} i-\gamma_{2} i, \xi_{2}=\frac{\xi_{1}^{2}\left(\gamma_{2}-4 \alpha_{2}^{3}\right)}{4\left(3 \alpha_{2}^{2} \alpha_{1} i+3 \alpha_{1}^{3} i+3 \alpha_{1}^{2} \alpha_{2}+\gamma_{2}-\alpha_{2}^{3}\right)},
$$

where $\alpha_{1}, \alpha_{2}, \beta_{2}, \gamma_{2}, \xi_{1}$ are free real constants. Substituting Eq. (9) into Eq. (4) and taking $\alpha_{2}=A_{2} i, \beta_{2}=B_{2} i, \gamma_{2}=$ $C_{2} i$ and $M>0$, we have

$$
f_{2}=2 \sqrt{M} \cosh \left(\alpha_{1} x-B_{2} y+E_{2} t+\frac{1}{2} \ln (M)\right)+\xi_{1} \cosh \left(A_{2} x+B_{2} y+C_{2} t\right),
$$

where $E_{2}=3 \alpha_{1}^{2} A_{2}-3 A_{2}^{2} \alpha_{1}-\alpha_{1}^{3}+A_{2}^{3}+C_{2}, M=\frac{\xi_{1}^{2}\left(4 \alpha_{2}^{3}+\gamma_{2}\right)}{4\left(3 \alpha_{1}^{2} A_{2}-3 \alpha_{1} A_{2}^{2}+3 \alpha_{1}^{3}+A_{2}^{3}+\gamma_{2}\right)}$. Substituting Eq. (10) into Eq. (2), we obtain the cross-kink two-solitary wave solutions for BLMP equation as follows(see Fig (b))

$$
\varphi_{2}=-\frac{2\left(2 \alpha_{1} \sqrt{M} \sinh \left(\theta+\frac{1}{2} \ln (M)\right)+\xi_{1} A_{2} \sinh (\tau)\right)}{2 \sqrt{M} \cosh \left(\theta+\frac{1}{2} \ln (M)\right)+\xi_{1} \cosh (\tau)},
$$

where $\theta=\alpha_{1} x-B_{2} y+E_{2} t, \tau=A_{2} x+B_{2} y+C_{2} t$.

\section{Breather-type Kink and Kink Three Wave Solutions}

If we choose extended homoclinic test funtion as

$$
f=e^{-\theta}+\xi_{1} \cos (\tau)+\xi_{2} \sinh (\omega)+\xi_{3} e^{\theta},
$$


where $\theta=\alpha_{1} x+\beta_{1} y+\gamma_{1} t, \tau=\alpha_{2} x+\beta_{2} y+\gamma_{2} t, \omega=\alpha_{3} x+\beta_{3} y+\gamma_{3} t$ and $\alpha_{i}, \beta_{i}, \gamma_{i}, \xi_{i}(i=1,2,3)$ are some constants to be determined later. Substituting Eq. (12) into Eqs. (3), and equating all the coefficients of different powers of $e^{\theta}, e^{-\theta}, \sin (\tau), \cos (\tau), \sinh (\omega), \cosh (\omega)$ and constant term to zero yields a set of algebraic equations:

$$
\left\{\begin{array}{l}
\xi_{1}\left(\alpha_{2}^{3} \beta_{2}-\alpha_{1}^{3} \beta_{1}-\beta_{2} \gamma_{2}-3 \alpha_{1}^{2} \alpha_{2} \beta_{2}+\beta_{1} \gamma_{1}-3 \alpha_{2}^{2} \alpha_{1} \beta_{1}\right)=0 \\
\xi_{2}\left(\alpha_{3}^{3} \beta_{1}+\beta_{1} \gamma_{3}+\beta_{3} \gamma_{1}+3 \alpha_{1}^{2} \alpha_{3} \beta_{1}+\alpha_{1}^{3} \beta_{3}+3 \alpha_{1} \alpha_{3}^{2} \beta_{3}\right)=0 \\
\xi_{2}\left(\beta_{3} \gamma_{3}+3 \alpha_{3}^{2} \alpha_{1} \beta_{1}+\beta_{1} \gamma_{1}+\alpha_{3}^{3} \beta_{3}+3 \alpha_{1}^{2} \alpha_{3} \beta_{3}+\alpha_{1}^{3} \beta_{1}\right)=0 \\
\xi_{1} \xi_{2}\left(\alpha_{2}^{3} \beta_{2}+\alpha_{3}^{3} \beta_{3}-3 \alpha_{2} \alpha_{3}^{2} \beta_{2}+\beta_{3} \gamma_{3}-\beta_{2} \gamma_{2}-3 \alpha_{2}^{2} \alpha_{3} \beta_{3}\right)=0 \\
\xi_{1} \xi_{3}\left(-\alpha_{2}^{3} \beta_{1}+\alpha_{1}^{3} \beta_{2}+\beta_{1} \gamma_{2}+\beta_{2} \gamma_{1}+3 \alpha_{1}^{2} \alpha_{2} \beta_{1}-3 \alpha_{1} \alpha_{2}^{2} \beta_{2}\right)=0 \\
\xi_{1} \xi_{3}\left(\alpha_{2}^{3} \beta_{2}+\alpha_{1}^{3} \beta_{1}-\beta_{2} \gamma_{2}-3 \alpha_{1}^{2} \alpha_{2} \beta_{2}+\beta_{1} \gamma_{1}-3 \alpha_{2}^{2} \alpha_{1} \beta_{1}\right)=0 \\
\xi_{2} \xi_{3}\left(\beta_{3} \gamma_{3}+3 \alpha_{3}^{2} \alpha_{1} \beta_{1}+\beta_{1} \gamma_{1}+\alpha_{3}^{3} \beta_{3}+3 \alpha_{1}^{2} \alpha_{3} \beta_{3}+\alpha_{1}^{3} \beta_{1}\right)=0 \\
\xi_{1}\left(-\alpha_{2}^{3} \beta_{1}+\alpha_{1}^{3} \beta_{2}+\beta_{1} \gamma_{2}+\beta_{2} \gamma_{1}+3 \alpha_{1}^{2} \alpha_{2} \beta_{1}-3 \alpha_{1} \alpha_{2}^{2} \beta_{2}\right)=0 \\
\xi_{1} \xi_{2}\left(-\beta_{3} \gamma_{2}+3 \alpha_{2}^{2} \alpha_{3} \beta_{2}+\alpha_{2}^{3} \beta_{3}-3 \alpha_{3}^{2} \alpha_{2} \beta_{3}-\alpha_{3}^{3} \beta_{2}-\beta_{2} \gamma_{3}\right)=0 \\
\xi_{2} \xi_{3}\left(\alpha_{3}^{3} \beta_{1}+\beta_{1} \gamma_{3}+\beta_{3} \gamma_{1}+3 \alpha_{1}^{2} \alpha_{3} \beta_{1}+\alpha_{1}^{3} \beta_{3}+3 \alpha_{1} \alpha_{3}^{2} \beta_{3}\right)=0 \\
4 \xi_{1}^{2} \alpha_{2}^{3} \beta_{2}-\xi_{1}^{2} \beta_{2} \gamma_{2}+16 \xi_{3} \alpha_{1}^{3} \beta_{1}+4 \xi_{3} \beta_{1} \gamma_{1}+4 \xi_{2}^{2} \alpha_{3}^{3} \beta_{3}+\xi_{2}^{2} \beta_{3} \gamma_{3}=0,
\end{array}\right.
$$

Solving the system Eqs.(13), we obtain the following cases

Case(III):

$$
\alpha_{3}=-\alpha_{1}, \beta_{3}=\beta_{1}, \gamma_{1}=\alpha_{1}\left(3 \alpha_{2}^{2}-\alpha_{1}^{2}\right), \gamma_{2}=\alpha_{2}\left(\alpha_{2}^{2}-3 \alpha_{1}^{2}\right), \gamma_{3}=\alpha_{1}\left(\alpha_{1}^{2}-3 \alpha_{2}^{2}\right), \xi_{3}=\frac{\alpha_{1} \beta_{1} \xi_{2}^{2}-\alpha_{2} \beta_{2} \xi_{1}^{2}}{4 \alpha_{1} \beta_{1}},
$$

where $\alpha_{1}, \alpha_{2}, \beta_{1}, \beta_{2}, \xi_{1}, \xi_{2}$ are free real constants. Substituting Eq.(14) into Eq.(4) and and taking $M>0$, we have

$$
f_{3}=2 \sqrt{M} \cosh \left(\alpha_{1} x+\beta_{1} y-E_{3} t+\frac{1}{2} \ln (M)\right)+\xi_{1} \cosh \left(\alpha_{2} x+\beta_{2} y+I_{3} t\right)-\xi_{2} \sinh \left(\alpha_{1} x-\beta_{1} y-E_{3} t\right),
$$

where $E_{3}=\alpha_{1}\left(3 \alpha_{2}^{2}-\alpha_{1}^{2}\right), I_{3}=\alpha_{2}\left(\alpha_{2}^{2}-3 \alpha_{1}^{2}\right), M=\frac{\alpha_{1} \beta_{1} \xi_{2}^{2}-\alpha_{2} \beta_{2} \xi_{1}^{2}}{4 \alpha_{1} \beta_{1}}$. Substituting Eq. (15) into Eq. (2), we obtain the breather-type kink three wave solutions for BLMP equation as follows(see Fig (c))

$$
\varphi_{3}=-\frac{2\left(2 \alpha_{1} \sqrt{M} \sinh \left(\theta+\frac{1}{2} \ln (M)\right)-\xi_{1} \alpha_{2} \sin (\tau)-\xi_{2} \alpha_{1} \cosh (\omega)\right)}{2 \sqrt{M} \cosh \left(\theta+\frac{1}{2} \ln (M)\right)+\xi_{1} \cos (\tau)-\xi_{2} \sinh (\omega)},
$$

where $\theta=\alpha_{1} x+\beta_{1} y-E_{3} t, \tau=\alpha_{2} x+\beta_{2} y+I_{3} t, \omega=\alpha_{1} x-\beta_{1} y-E_{3} t$. The solution represented by Eq.(14) is a breather-type kink three wave which has breather effect when wave along with straight line $\alpha_{2} x+\beta_{2} y+I_{3} t=d$ and also is a two-solitary wave as $\alpha_{2} x+\beta_{2} y+I_{3} t=d$, where $d$ is a constant.

Case(IV):

$$
\begin{aligned}
& \alpha_{2}=\left(2 \alpha_{1}-\alpha_{3}\right) i, \beta_{1}=\beta_{2} i, \beta_{3}=-\beta_{2} i, \gamma_{1}=-4 \alpha_{1}^{3}, \gamma_{2}=-\left(5 \alpha_{1}^{3}-3 \alpha_{1}^{2} \alpha_{3}+3 \alpha_{3}^{2} \alpha_{1}-\alpha_{3}^{3}\right) i, \\
& \gamma_{3}=3 \alpha_{3}^{2}-\alpha_{3}^{3}-3 \alpha_{1}^{2} \alpha_{3}-3 \alpha_{1}^{3}, \xi_{1}=\frac{\xi_{2}\left(\alpha_{1}+\alpha_{3}\right)}{\alpha_{3}-3 \alpha_{1}} .
\end{aligned}
$$

where $\alpha_{1}, \alpha_{3}, \beta_{2}, \gamma_{2}, \xi_{2}, \xi_{3}$ are free real constants. Substituting Eq.(15) into Eq.(4) and taking $\delta_{3}>0$, we have

$$
f_{4}=2 \sqrt{\xi_{3}} \cosh \left(\alpha_{1} x-B_{2} y-4 \alpha_{1}^{3} t+\frac{1}{2} \ln \left(\xi_{3}\right)\right)+M \cosh \left(E_{4} x+B_{2} y+I_{4} t\right)+\xi_{2} \sinh \left(\alpha_{3} x+B_{2} y+G_{4} t\right),
$$

where $M=\frac{\xi_{2}\left(\alpha_{1}+\alpha_{3}\right)}{\alpha_{3}-3 \alpha_{1}}, E_{4}=2 \alpha_{1}-\alpha_{3}, I_{4}=3 \alpha_{1}^{2} \alpha_{3}+\alpha_{3}^{3}-5 \alpha_{1}^{3}-3 \alpha_{3}^{2} \alpha_{1}, G_{4}=3 \alpha_{3}^{2}-\alpha_{3}^{3}-3 \alpha_{1}^{2} \alpha_{3}-3 \alpha_{1}^{3}$. Substituting Eq. (16) into Eq. (2), we obtain the kink three soliton solutions for BLMP equation as follows

$$
\varphi_{4}=-\frac{2\left(2 \alpha_{1} \sqrt{\xi_{3}} \sinh \left(\theta+\frac{1}{2} \ln \left(\xi_{3}\right)\right)-M\left(\alpha_{3}-2 \alpha_{1}\right) \sinh (\tau)+\xi_{2} \alpha_{3} \cosh (\omega)\right)}{2 \sqrt{\xi_{3}} \cosh \left(\theta+\frac{1}{2} \ln \left(\xi_{3}\right)\right)+M \cosh (\tau)+\xi_{2} \sinh (\omega)},
$$

where $\theta=\alpha_{1} x-B_{2} y-4 \alpha_{1}^{3} t, \tau=E_{4} x+B_{2} y+I_{4} t, \omega=\alpha_{3} x+B_{2} y+G_{4} t$.

\section{Conclusion}

In summary, successfully applying the extended homoclinic test method to the (2+1)-Dimensional Boiti-LeonManna-Pempinelli equation, we obtain new breather soliton and cross-kink soliton solutions. With the aid of Maple, this method provides a powerful mathematical tool to obtain exact solutions. The extended homoclinic test approach can also be applied to solve other types of higher dimensional integrable and non-integrable systems. 


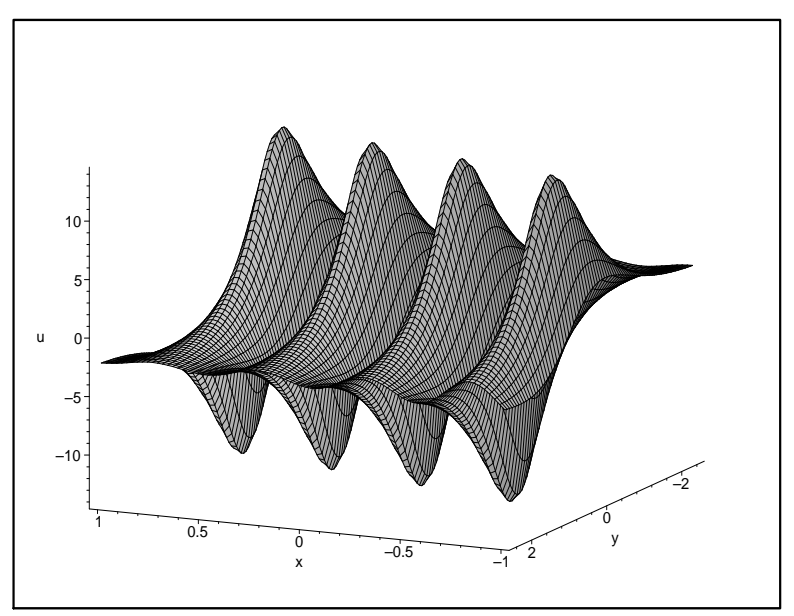

Figure(a). The figure of $\varphi_{1}$ as $\alpha_{1}=1, \beta_{1}=1.5$ $\beta_{2}=\frac{1}{2}, \xi_{1}=1, \xi_{2}=1, t=0$.

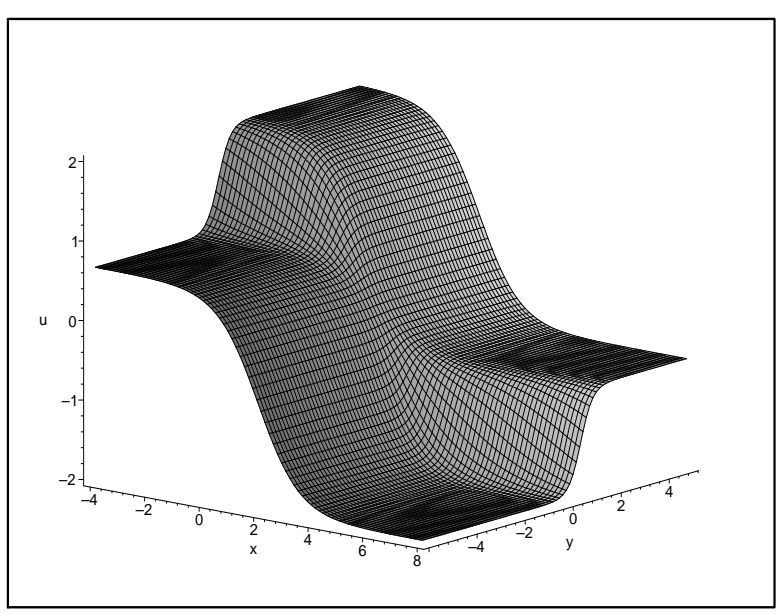

Fig(b). Figure (b). The figure of $\varphi_{2}$ as $\alpha_{1}=1, A_{2}=\frac{1}{3}$, $B_{2}=1.5, C_{2}=\frac{1}{2}, \xi_{1}=1, \xi_{2}=2, t=-1$.

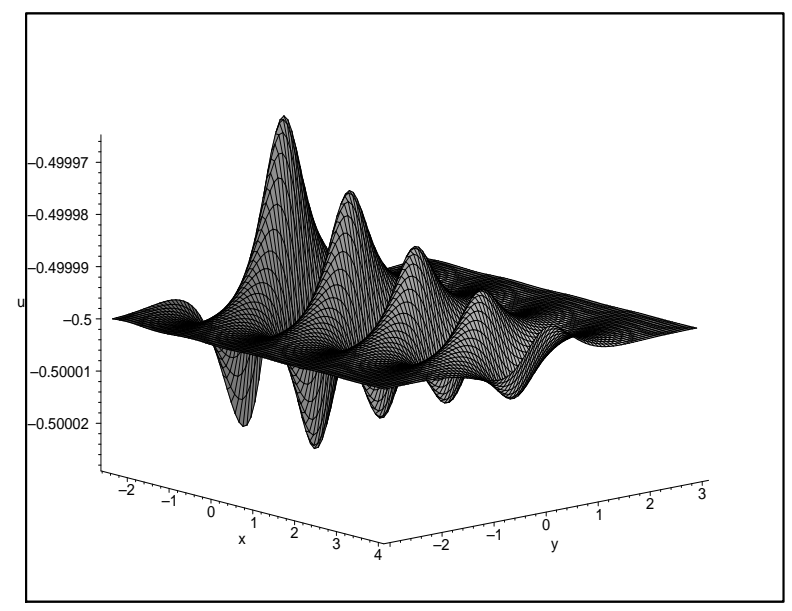

Figure (c).The figure of $\varphi_{3}$ as $\xi_{1}=\frac{1}{2}, \xi_{2}=1, \alpha_{1}=\frac{1}{4}, \beta_{1}=2, \alpha_{2}=4, \beta_{2}=2, t=1$.

\section{References}

Aiyer, R. N., Fuchssteiner, B. \& Oevel, W. (1986). Solitons and discrete eigenfunctions of the recursion operator of non-linear evolution equations: the Caudrey-Dodd-Gibbon-Sawada-Kotera equations. J. Phys. A: Math. General, 19, 3755-3770.

Dai, Z. D., Huang, J., Jiang, M. R. \& Wang, S. H. (2005). Homoclinic orbits and periodic solitons for Boussinesq equation with even constraint. Chaos Soliton and Fractals, 26, 1189-1194.

Dai, Z. D., Li, S. L., Dai, Q. Y. \& Huang, J. (2007). Singular periodic soliton solutions and resonance for the Kadomtsev-Petviashvili equation. Chaos Soliton and Fractals, 34, 1148-1153.

Dai, Z. D., Liu, J. \& Liu, Z. J. (2010). Exact periodic kink-wave and degenerative soliton solutions for potential Kadomtsev- Petviashvili equation. Commum. Nonlinear Sci. Numer. Simulat., 15(9), 2331-2336.

Dai, Z. D., Liu, J., Zeng, X. P. \& Liu, Z. J. (2008). Periodic kink-wave and kinky periodic-wave solutions for the Jimbo-Miwa equation. Phys Lett A, 372, 5984-5986.

He, J. H. (2006). New interpretation of homotopy perturbation method. Int. J. Mod. Phys. B, 20, 2561-2568. 
Luo, L. (2011). New exact solutions and Bäcklund transformation for Boiti-Leon-Manna-Pempinelli equation. Physics Letters A, 375, 1059-1063.

Ma, W. X. \& Fan ,E. G. (2011). Linear superposition principle applying to Hirota bilinear equations. Computers and Mathematics with Applications, 61, 950-959.

Ma, W. X., Alrazi, A., \& Asaad, M. G. (2011). Wronskian and Grammian solutions to a (3+1)-dimensional generalized KP equation. Appl. Math. Comp., 217,10016-10023.

Mabrouk, S., \& Kassem, M. (2014). Group similarity solutions of (2+1) Boiti-Leon-Manna-Pempinelli Lax pair. Ain Shams Engineering Journal, 5, 227-235.

Wang, C. J., Dai, Z. D. \& Liang, L.(2010). Exact three-wave solution for higher dimensional KdV-type equation. Applied Mathematics and Computation, 216, 501-505.

Weiss J. (1984). On classes of integrable systems and the Painlev é property. J. Math. Phys., 25(1),13-24.

Xu, Z. H., \& Xian, D. Q. (2010). New periodic solitary-wave solutions for the Benjiamin Ono equation. Applied Mathematics and Computation, 215, 4439-4442.

\section{Copyrights}

Copyright for this article is retained by the author(s), with first publication rights granted to the journal.

This is an open-access article distributed under the terms and conditions of the Creative Commons Attribution license (http://creativecommons.org/licenses/by/3.0/). 